\title{
Light-emitting Devices Fabricated with CdSe Nano-crystals Coated on an InGaN/GaN Quantum-well Structure for Polychromatic Generation
}

\author{
Dong-Ming Yeh, Chih-Feng Lu, Chi-Feng Huang, Tsung-Yi Tang, Horng-Shyang Chen, \\ Jian-Jang Huang, and C. C. Yang \\ Graduate Institute of Electro-Optical Engineering and Department of Electrical Engineering, \\ National Taiwan University, 1, Roosevelt Road, Sec. 4, Taipei, Taiwan, R.O.C. \\ (phone) 886-2-23657624 (fax) 886-2-23652637（e-mail) ccy@cc.ee.ntu.edu.tw
} Chih-Min Chuang and Wei-Fang Su

Department of Material Science and Engineering, National Taiwan University, Taipei, Taiwan.

\begin{abstract}
:
By coating CdSe nano-crystals on an $\mathrm{InGaN} / \mathrm{GaN}$ quantum well structure, polychromatic LEDs emitting blue and red lights have been implemented. The blue photons emitted by the quantum well structure are absorbed by the CdSe nano-crystals for emitting red light. By fabricating holes on the quantum well structure for filling up the nano-crystal solution, the red emission efficiency can be improved.

\section{Introduction}

The techniques of color mixing for polychromatic display and white-light generation are important for the developments of micro-display and solid-state lighting. For polychromatic display, multi-element light-emitting diode (LED) arrays of different colors are widely used. To implement white-light generation, polymer phosphors are usually placed on either blue or UV LEDs for generating complementary colors. However, the use of phosphors normally results in poor efficiency and difficulty in packaging.
\end{abstract}

\section{Device Design}

In this research, we adopt an all-semiconductor approach for implementing polychromatic generation. We coat CdSe nano-crystals on an InGaN/GaN multiple quantum-well (QW) structure. The QW structure emits blue light or blue/green light. The CdSe nano-crystals absorb the blue/green light from the QW structure and emits red light. The absorption and emission wavelengths of the CdSe nano-crystals depend on the size of $\mathrm{CdSe}$ nano-crystal and its coating of $\mathrm{ZnS}$. To enhance the energy transfer efficiency, the photon emission wavelength of the InGaN/GaN QW structure and the major absorption spectral range of the $\mathrm{CdSe}$ nano-crystals must be matched. Also, to implement the required color mixture, the emission wavelengths and intensities from the QWs and nano-crystals must be well adjusted. For these purposes, the QW composition and well width and the size of CdSe nano-crystal need to be carefully designed.

\section{Experimental Procedures}

In our current implementation, the CdSe nano-crystals $(4.5 \mathrm{~nm}$ in size with a $\mathrm{ZnS}$ coating of $0.2 \mathrm{~nm}$ in thickness) have a reasonably strong absorption coefficient around $450 \mathrm{~nm}$ (see Fig. 1 for the photoluminescence excitation data) and emit light at $599 \mathrm{~nm}$. Therefore, we can mix the blue and red colors. To enhance the energy transfer efficiency from blue to red, we fabricate holes with the diameters ranging from 50 to 300 microns on the top of the LED for filling up with the CdSe nano-crystal solution (see Fig. 2). Fig. 3 shows the SEM image of the device top view with $70 \mu \mathrm{m}$ holes. The nano-crystals in the holes can absorb the emitted blue photons from the QW structure more easily, leading to stronger red emission. Besides the mixing of blue and red colors, the green color can be added by using an epitaxial sample of two diodes, which is grown with two sets of QW for blue and green emissions.

\section{Results}

Fig. 4 shows the electroluminescence spectra of the fabricated LEDs with different hole sizes. The results of the standard device are also shown for comparison. Here, the standard device results in the strongest blue intensity and weakest red intensity. The device with $10 \mu \mathrm{m}$ holes provides the strongest red emission and the weakest blue emission. The relative red emission intensity decreases with increasing hole size. Compared with the standard device, the device of $10 \mu \mathrm{m}$ holes shows a $30 \%$ improvement in red emission intensity. It is believed that by decreasing the hole size, the red emission efficiency can be further improved. When the hole 
size is smaller than the wavelength, the improvement will be much more significant.

\section{Conclusions}

In conclusion, we have fabricated polychromatic LEDs emitting blue and red lights by coating CdSe nano-crystals on an InGaN/GaN quantum well structure. The blue photons emitted by the quantum well structure were absorbed by the $\mathrm{CdSe}$ nano-crystals for emitting red light. By fabricating holes on the quantum well structure for filling up the nano-crystal solution, the red emission efficiency has been improved.

\section{Acknowledgement:}

This research was supported by National Science Council, The Republic of China, and by US Air Force.

\section{Reference:}

1. Marc Achermann, Melissa A. Petruska, Simon Kos, Darryl L. Smith, Daniel D. Koleske, and Victor

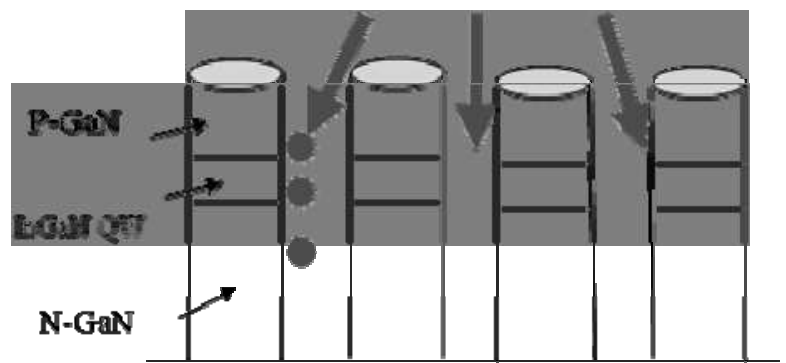

Fig. 2 Holes are fabricated on the top of the LED for filling up with CdSe nano-crystal solution.
I. Klimov, Nature 429, 642-646 (2004).

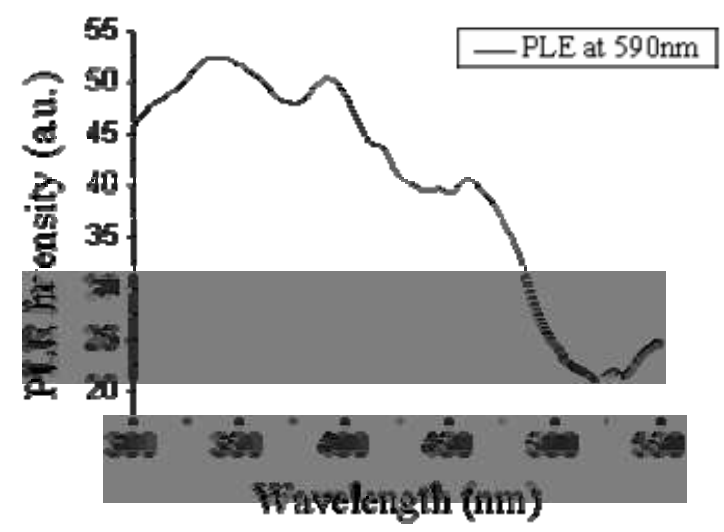

Fig. 1 Photoluminescence excitation spectrum of the nano-crystals.

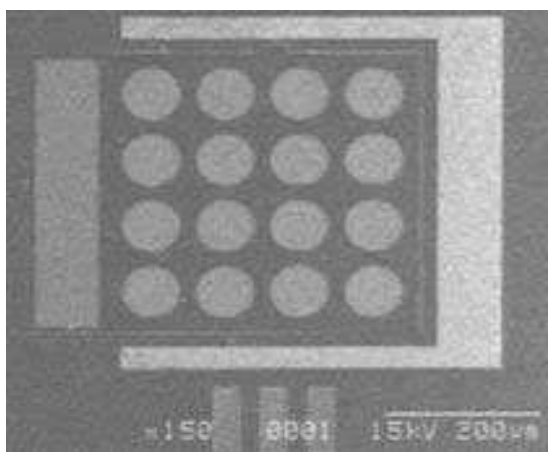

Fig. 3 SEM image of the device with $70-\mu \mathrm{m}$ holes.

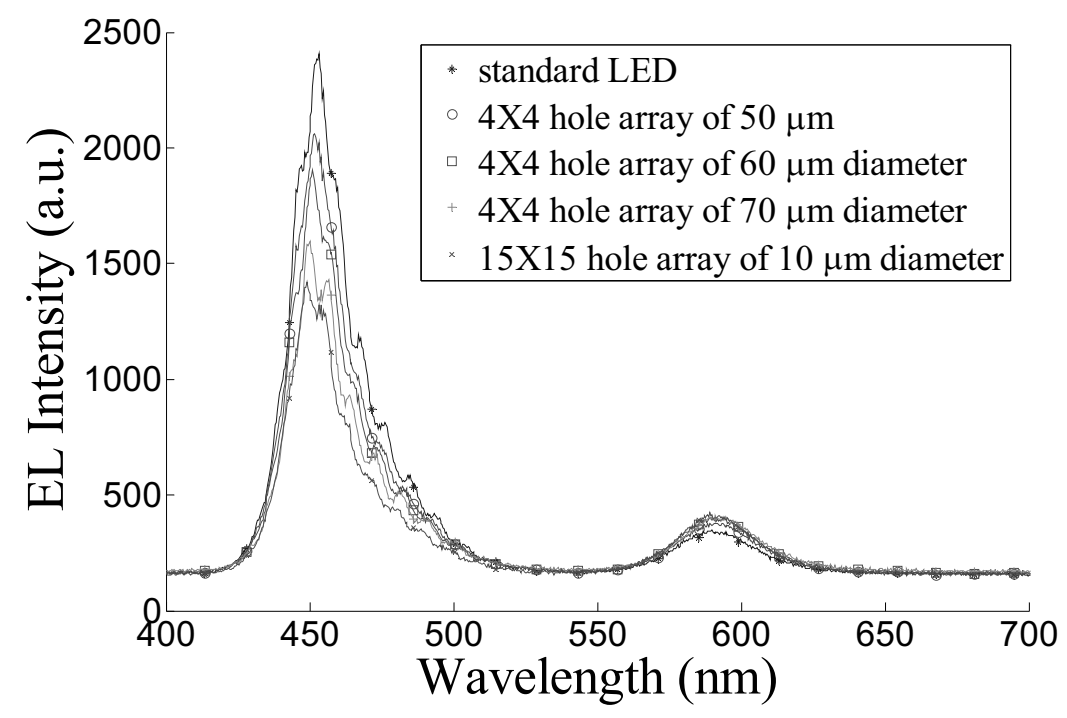

Fig. 4 Electroluminescence spectra of the fabricated LEDs with different hole sizes. 